\title{
Spin Liquid State and Topological Structural Defects in Hexagonal $\mathrm{TbInO}_{3}$
}

\author{
Jaewook Kim, ${ }^{1}$ Xueyun Wang, ${ }^{1}$ Fei-Ting Huang, ${ }^{1}$ Yazhong Wang, ${ }^{1}$ Xiaochen Fang, ${ }^{1}$ Xuan Luo, ${ }^{2}$ Y. Li, ${ }^{1,3}$ \\ Meixia Wu, ${ }^{1,4}$ S. Mori, ${ }^{5}$ D. Kwok, ${ }^{1}$ Eun Deok Mun, ${ }^{6,7}$ V. S. Zapf, ${ }^{6}$ and Sang-Wook Cheong ${ }^{1,2}$ \\ ${ }^{1}$ Rutgers Center for Emergent Materials and Department of Physics and Astronomy, \\ Rutgers University, Piscataway, New Jersey 08854, USA \\ ${ }^{2}$ Laboratory for Pohang Emergent Materials and Max Plank POSTECH Center for Complex Phase Materials, \\ Pohang University of Science and Technology, Pohang 790-784, Republic of Korea \\ ${ }^{3}$ State Key Laboratory of Crystal Materials, Shandong University, \\ Jinan, Shandong, People's Republic of China \\ ${ }^{4}$ School of Materials Science and Engineering, South China University of Technology, \\ Guangzhou, People's Republic of China \\ ${ }^{5}$ Department of Materials Science, Osaka Prefecture University, Sakai, Osaka 599-8531, Japan \\ ${ }^{6}$ National High Magnetic Field Laboratory, Los Alamos National Laboratory, \\ Los Alamos, New Mexico 87545, USA \\ ${ }^{7}$ Department of Physics, Simon Fraser University, Burnaby, British Columbia, V5A 1S6, Canada
}

(Received 6 August 2018; revised manuscript received 17 April 2019; published 9 July 2019)

\begin{abstract}
We report the coexistence of ferroelectricity and a spin liquid state in hexagonal $\mathrm{TbInO}_{3}$ with quasitwo-dimensional triangular spin lattice. Geometrical ferroelectricity associated with In trimerization accompanies topological ferroelectric structural defects. Magnetic susceptibility data show in-plane magnetic anisotropy of $\mathrm{Tb}$ spins without any long-range order above $1.8 \mathrm{~K}$, and we also confirm no trace of any phase transition down to $0.15 \mathrm{~K}$ from a specific heat measurement, which indicates that this system is highly frustrated and may host a spin liquid ground state. By analyzing the Schottky anomaly in the specific heat results, we propose a model where crystal-field levels are different in each $\mathrm{Tb}$ sites, and only one of them has a magnetic ground state and forms a unique honeycomb spin lattice. These observations put forward an interesting possibility where spin liquid and ferroelectric behaviors coexist, and the atomically sharp ferroelectric domain walls may host new magnetic edge states or local spin excitations.
\end{abstract}

DOI: 10.1103/PhysRevX.9.031005

Subject Areas: Condensed Matter Physics, Magnetism, Materials Science

\section{INTRODUCTION}

Geometrically frustrated magnetic systems provide a plethora of exotic ground states in condensed matter physics [1]. Competition between underlying interactions leads to highly degenerate ground states at low temperatures that often involve unconventional quantum phenomena. A simplified but widely studied example of such states is the two-dimensional $(d)$ triangular lattice (TL) with antiferromagnetic (AFM) interactions [2]. In the case of an Ising-type spin system, it is well established that the system does not form any long-range order (LRO) even at a zero temperature $(T)$ with large residual entropy $[3,4]$, while a classical Heisenberg-type spin system undergoes a LRO at

Published by the American Physical Society under the terms of the Creative Commons Attribution 4.0 International license. Further distribution of this work must maintain attribution to the author(s) and the published article's title, journal citation, and DOI.
$T=0$ to a coplanar $120^{\circ}$ structure $[5,6]$. The special case where spins are $S=1 / 2$ in a triangular lattice was extensively studied after the seminal prediction by Anderson [7] of a "quantum spin liquid" where a strong quantum fluctuation prevents any LRO down to $T=0$ [8-11]. However, recent works confirm that it undergoes a LRO at $T=0$ with a similar structure to its classical counterpart [12-14]. Nevertheless, the proposed concept of a spin liquid still remains elusive and has motivated further research on other geometrically frustrated systems such as pyrochlore and kagome lattice systems [15-17].

Recent efforts, however, have rejuvenated the $2 d$-TL systems as a platform to realize a spin liquid state with an emphasis on large spin-orbit coupling from rare-earth $(R)$ ions. In these systems, despite the large magnetic moment, spin-orbit coupling and local crystal fields (CFs) together cast the rare-earth spins into doublet ground states [18-23], and the localized nature of $4 f$ orbitals limits the spatial extent of the exchange interactions perhaps only to the nearest-neighbor interaction. A prototypical example may 

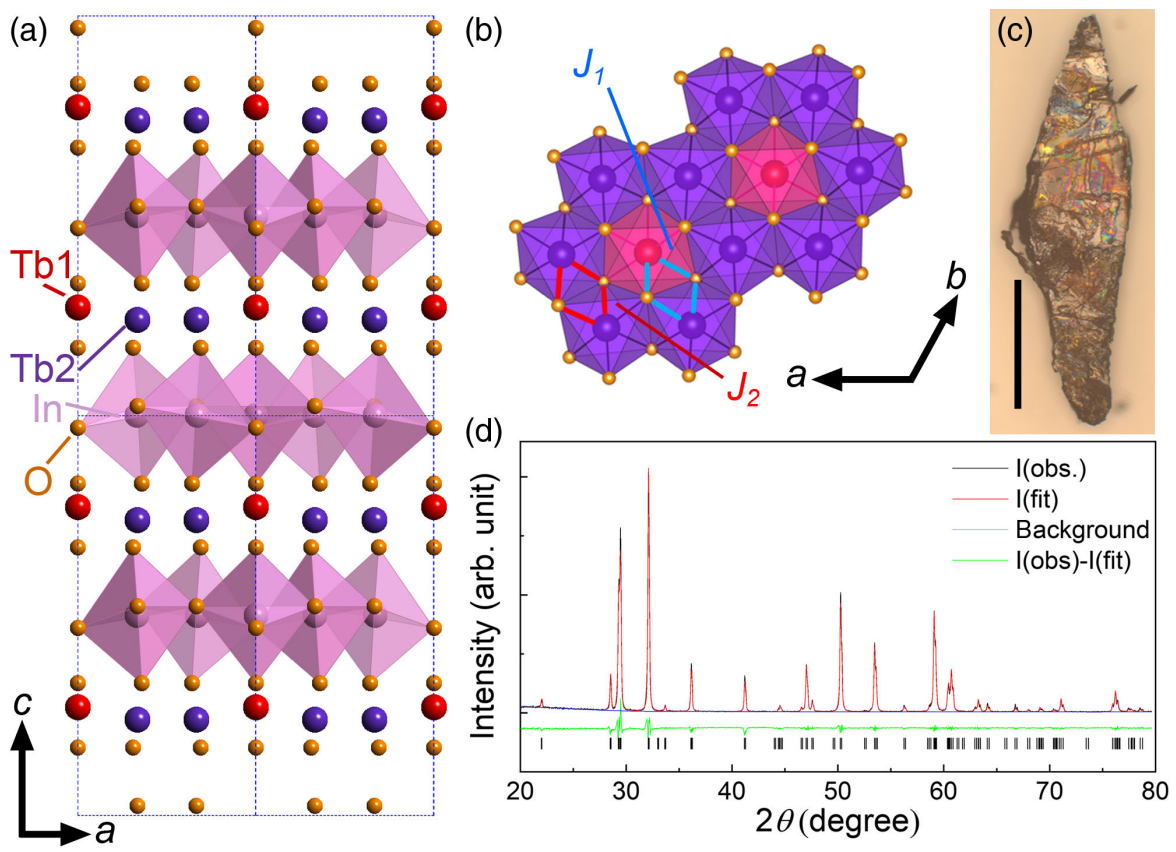

FIG. 1. (a) Crystal structure of $\mathrm{TbInO}_{3}$. Tb ions with two different sites ( $\mathrm{Tb} 1$ and $\mathrm{Tb} 2$ ), In, and $\mathrm{O}$ ions are shown as red, purple, violet, and orange spheres, respectively. In ions are located inside the oxygen trigonal bipyramids. (b) In-plane structure of the honeycomb $\mathrm{TbO}_{6}$ polyhedra network. Red and purple polyhedra enclose Tb1 and Tb2 ions, respectively. $J_{1}$ and $J_{2}$ denote nearest-neighbor interaction paths between Tb1-Tb2 and Tb2-Tb2 spins, respectively. (c) Photograph of a cleaved single crystal. The bottom scale is $0.5 \mathrm{~mm}$. (d) X-ray diffraction data taken at room temperature and Rietveld refinement on a powder sample. Black, red, blue, and green lines are the observed intensity, the fitted intensity, the background, and the difference between the observed and fitted intensity, respectively. Black markers indicate refined peak positions.

be $\mathrm{YbMgGaO}_{4}$, which forms a perfect $2 d$-TL with $\mathrm{Yb}^{3+}$ ions but remains magnetically disordered down to $60 \mathrm{mK}$ $[18,19]$. Although it has been recently argued that $\mathrm{Mg} / \mathrm{Ga}$ site disorder plays an important role in suppressing LRO in $\mathrm{YbMgGaO}_{4}$ [24,25], large spin-orbit coupling of rareearth ions, in general, can lead to bond-dependent exchange coupling, similar to a Kitaev-type interaction [19-22]. More recently, a number of other systems have been proposed theoretically or studied experimentally in polycrystalline forms of a rare-earth-based TL, expanding the search for unconventional physics in this class of materials [26].

An intriguing prediction for spin liquids that has been less studied experimentally is its nontrivial edge states. In general, long-range entanglement in quantum spin liquid systems can be a source of nontrivial edge states exhibiting perfect heat conduction or quantized spin-Hall conductance [27-30], which can be compared, for example, to the quantum Hall effect in $2 d$ electronic systems [31]. Recently, a quantized thermal Hall effect was reported in a Kitaev spin liquid $\alpha-\mathrm{RuCl}_{3}$ [30]. In principle, such nontrivial edge states are not limited to the sample boundaries but also can exist at domain boundaries. For example, a domain boundary between $A B$ - and $B A$-stacked bilayer graphene forms a conducting channel [32], and a quantum anomalous Hall insulator can show chiral edge states at magnetic domain boundaries [33]. Nevertheless, domain boundary effects in spin liquids have not been studied to date mainly due to a lack of proper materials and experimental probes that can spatially resolve local transport properties [34].

In this work, we report the physical properties of the $2 d$-TL system $\mathrm{TbInO}_{3}$, which is isostructural to the hexagonal $R \mathrm{MnO}_{3}$ [35]. In contrast to magnetic LRO of Mn spins in $R \mathrm{MnO}_{3}$ systems, we find that $\mathrm{TbInO}_{3}$ does not show any magnetic phase transition above $T \geq 0.15 \mathrm{~K}$ with an in-plane magnetic anisotropy, suggesting that this system can have a spin liquid ground state. Our specific heat data show an unusual Schottky anomaly behavior that can be described by adopting different CF levels in two different crystallographic $\mathrm{Tb}$ sites. Our analysis suggests that only one of them ( $\mathrm{Tb} 2)$ has a magnetic ground state that forms an emergent $2 d$-honeycomb magnetic lattice, consistent with a recent report on this system studied by inelastic neutron scattering [36]. Furthermore, we show that $\mathrm{TbInO}_{3}$ is a ferroelectric with topological structural defects and $Z_{2} \times Z_{3}$ domains that form atomically sharp domain boundaries. Both magnetic and ferroelectric properties combined, our study provides an unprecedented opportunity where mesoscopic spin liquid states are enclosed by ferroelectric domain boundaries. In particular, domain boundaries in this system may host nontrivial magnetic excitations, analogous to those predicted for the Kitaev honeycomb lattice model with lattice defects [37-39]. 


\section{METHODS}

Polycrystalline $\mathrm{TbInO}_{3}$ is synthesized via a conventional solid-state reaction method in air. Powders of $\mathrm{Tb}_{4} \mathrm{O}_{7}$ and $\mathrm{In}_{2} \mathrm{O}_{3}$ with an appropriate molar ratio are mixed, ground, and then pelletized for high-temperature sintering. The pellets are sintered at $1280^{\circ} \mathrm{C}$ for $36 \mathrm{~h}$ with intermediate grindings. Single-crystalline $\mathrm{TbInO}_{3}$ is grown using a laserfloating-zone (LD-FZ-5-200-VPO-PC, Crystal Systems Incorporation) technique under a pressurized- $\mathrm{O}_{2}$ atmosphere with the growth speed of $10 \mathrm{~mm} / h$. Feed rods for the floating-zone growth are prepared in the same manner as the polycrystalline sample. The resultant crystal boule is $50 \mathrm{~mm}$ long and $7 \mathrm{~mm}$ in diameter and is transparent yellow brown in color [see the photo in Fig. 1(c)]. With X-ray (X'Pert ${ }^{3}$ Powder diffractometer, Panalytical) and Laue (Photonic Science) diffraction, we confirm that the single crystal is single phase over the whole length. The crystal boule can be cleaved along the $a b$ plane, and a cleaved crystal $2 \times 2 \times 0.5 \mathrm{~mm}^{3}$ in size is used for the magnetization measurement. Specimens for transmission electron microscope (TEM) studies are prepared by mechanical polishing, followed by Ar ion milling. Domain structures are studied using a TEM (JEOL-2010F) equipped with a 14-bit chargecoupled-device array detector. Imaging plates are also used to record dark-field images. All the Miller indices described in this paper are based on the $P 6_{3} / \mathrm{mmc}$ parent phase. Highangle annular dark-field (HAADF) scanning TEM (STEM) imaging and chemical mapping with an atomic-column resolution are carried out using a scanning TEM (JEOLARM200F) equipped with a spherical aberration Cs corrector in conjunction with energy-dispersive $\mathrm{x}$-ray spectroscopy. Piezoelectric force microscopy is performed at room temperature using a scanning probe microscopy controller (Nanoscope IIIa, Digital Instruments) and a lock-in amplifier (SR830, SRS). Electric polarization is measured by using a commercial ferroelectric tester (Premium II, Radiant). Magnetic susceptibility is measured using a superconducting quantum interferometer (MPMS, Quantum Design). Magnetization up to $60 \mathrm{~T}$ is measured with an induction magnetometry technique [40] using a capacitor-bank-driven pulse magnet at the National High Magnetic Field Laboratory pulsed-field facility at Los Alamos. The pulsed-field magnetization values are calibrated against measurements in a $14 \mathrm{~T}$ dc magnet using a vibrating sample magnetometer (PPMS, Quantum Design). Specific heat is measured in a ${ }^{3} \mathrm{He} /{ }^{4} \mathrm{He}$ dilution refrigerator (PPMS, Quantum Design) down to $0.15 \mathrm{~K}$ using a thermal relaxation method.

\section{RESULTS}

\section{A. Structural and ferroelectric properties}

The hexagonal $R \mathrm{InO}_{3}(R=\mathrm{Sm}, \mathrm{Eu}, \mathrm{Gd}, \mathrm{Tb}, \mathrm{Dy}, \mathrm{Ho}$, and $\mathrm{Y}$ ) system crystallizes in the $P 6_{3} \mathrm{~cm}$ space group $[35,41,42]$. It consists of alternating layers of In and $R$ ions along the $c$ axis [Fig. 1(a)]. $R$ ions form a triangular lattice, but there are two different sites within each layer: $2 \mathrm{a}$ and $4 \mathrm{~b}$ for $R 1$ and $R 2$ ions, respectively. $R 2$ sites form a honeycomb structure, and the $R 1$ sites sit at the center of each honeycomb cell. Nearest-neighbor $R 1-R 2$ or $R 2-R 2$ pairs are connected via two oxygen and form an edgesharing network of $R_{6}$ polyhedra [Fig. 1(b)]. $\mathrm{InO}_{5}$ polyhedrons are tilted in such a way to induce structural trimerization of In ions. The imbalanced population of upward and downward distorted $R$ ions gives rise to the ferroelectric polarization along the $c$ axis [Fig. 1(a)]. Figure 1(d) shows x-ray diffraction data, which are in good agreement with previous results [35,41]. From structural refinement, we find that the lattice constants at room temperature are $a=6.319(1) \AA \quad$ and $c=12.312(2) \AA$. Details of the refinement are given in Supplemental Material [43].

The origin of structural trimerization and ferroelectricity is the size mismatch between $R$ and In, which leads to three antiphases $(\alpha, \beta$, and $\gamma)[44,45]$. Because of a subsequent ionic displacement with a net electric dipole moment, each trimerization phase results in two different ferroelectric polarizations $[\operatorname{up}(+)$ and down $(-)$ polarization along the $c$ axis] [46,47], which is directly observed in a dark-field (DF) TEM image as shown in Fig. 2(a). Here, the structural antiphase and the ferroelectric phases conspire to form ferroelectric vortex domains $\left(Z_{2} \times Z_{3}\right.$ domains with $Z_{6}$ vortices [48]). The network of vortices is assigned with the domain configuration described above, which can be analyzed in terms of the graph theory [49]. These six trimerization antiphase and ferroelectric domains cycle around a merging point in two different domain configurations: $(\alpha+, \beta-, \gamma+, \alpha-, \beta+, \gamma-)$ and $(\alpha-, \beta+, \gamma-, \alpha+$, $\beta-, \gamma+$ ), which can be viewed as a vortex and an antivortex, as the red and blue arrows indicate in Fig. 2(a), respectively. The HAADF-STEM image shows two regions with opposite ferroelectric polarization directions, which are displayed in Fig. 2(b). The dashed lines mark the boundaries between the up- and down-polarized regions. Note that the yellow and green dashed lines show transverse domain walls with $1 / 3$ and $2 / 3$ unit cell shifts, respectively.

The ferroelectric nature of the topological vortex domains is also supported by Piezoelectric force microscopy (PFM). Figure 2(c) shows a PFM image on the $a b$ plane of a single crystal. In contrast to more-or-less equal areal distribution of $+/-$ domains (type I) in the polycrystalline sample [Fig. 2(a)], single-crystal results show that one type of polarity is more favored than the other (type II) [50]. This behavior is likely due to a chemical, strain gradient, or self-poling effect [51] possibly originating from a large thermal gradient that exists during crystal growth using a laser-floating-zone technique. We also obtain ferroelectric hysteresis loops on single crystals with remnant polarization as high as approximately $0.9 \mu \mathrm{C} / \mathrm{cm}^{2}$ at $T=77 \mathrm{~K}$, as shown in Fig. 2(d). This value is smaller than that of $R \mathrm{MnO}_{3}$ systems (e.g., $\mathrm{YMnO}_{3} \sim 5.5 \mu C / \mathrm{cm}^{2}$ ) 

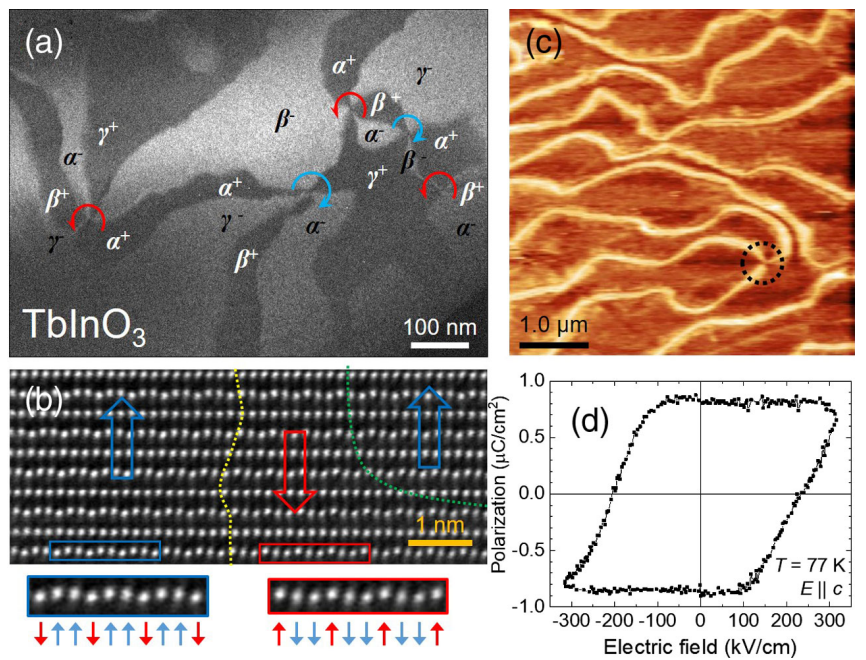

FIG. 2. (a) DF TEM images of polycrystalline $\mathrm{TbInO}_{3}$ taken under the Friedel pair-breaking condition. The network of vortices with assigned phases $(\alpha, \beta, \gamma,+,-)$ is depicted. (b) HAADF STEM image of polycrystalline $\mathrm{TbInO}_{3}$ with two different polarized regions (blue- and red-boxed regions). The bottom row shows enlarged images and two different domain walls (yellow and green dotted lines). (c) Out-of-plane contrast PFM image of a single crystal within the $a b$ plane. A topological vortex is located inside the dashed circle. (d) Electric polarization along the $c$ axis as a function of the applied electric field of a single crystal measured at $77 \mathrm{~K}$.
[52] and the theoretically predicted value $\left(10 \mu C / \mathrm{cm}^{2}\right)$ [42], which may be due to incomplete poling, probably associated with the existing chemical or strain gradient.

\section{B. Magnetic properties}

Figure 3(a) shows the $\chi(T)$ curves of polycrystals and single crystals down to $1.8 \mathrm{~K}$, measured in $\mu_{0} H=0.2 \mathrm{~T}$ after zero-magnetic-field cooling. The average value of single-crystal data, after considering crystalline anisotropy $\left(\chi_{\text {average }}=2 / 3 \chi_{a b}+1 / 3 \chi_{c}\right.$, green solid line $)$, well matches that of the polycrystalline one, which demonstrates the high quality of our crystals. Inverse magnetic susceptibility $\left(\chi^{-1}\right)$ as a function of the temperature for the polycrystal follows the Curie-Weiss behavior [inset in Fig. 3(a)] down to $20 \mathrm{~K}$ with an effective moment of $9.77 \mu_{B} / \mathrm{Tb}^{3+}$ and Curie-Weiss temperature $\left(T_{\mathrm{CW}}\right)$ of $-20.6 \mathrm{~K}$. The obtained effective moment well matches the theoretical spin-only value of $\mathrm{Tb}^{3+}\left(4 f^{8}, 9.72 \mu_{B}\right)$. The single-crystal $\chi(T)$ curve is larger (smaller) than that of the polycrystal below approximately $200 \mathrm{~K}$ along the $a b$ plane ( $c$ axis), suggesting an in-plane magnetic anisotropy. Consequently, $\chi^{-1}(T)$ of a single crystal along two directions follows the Curie-Weiss behavior only above $200 \mathrm{~K}$ with $T_{\mathrm{CW}}$ of $-205 \mathrm{~K}$ and $12 \mathrm{~K}$ for the $a b$ plane and $c$ axis magnetic field, respectively. $\chi(T)$ curves do not show any hint of LRO down to $1.8 \mathrm{~K}$.
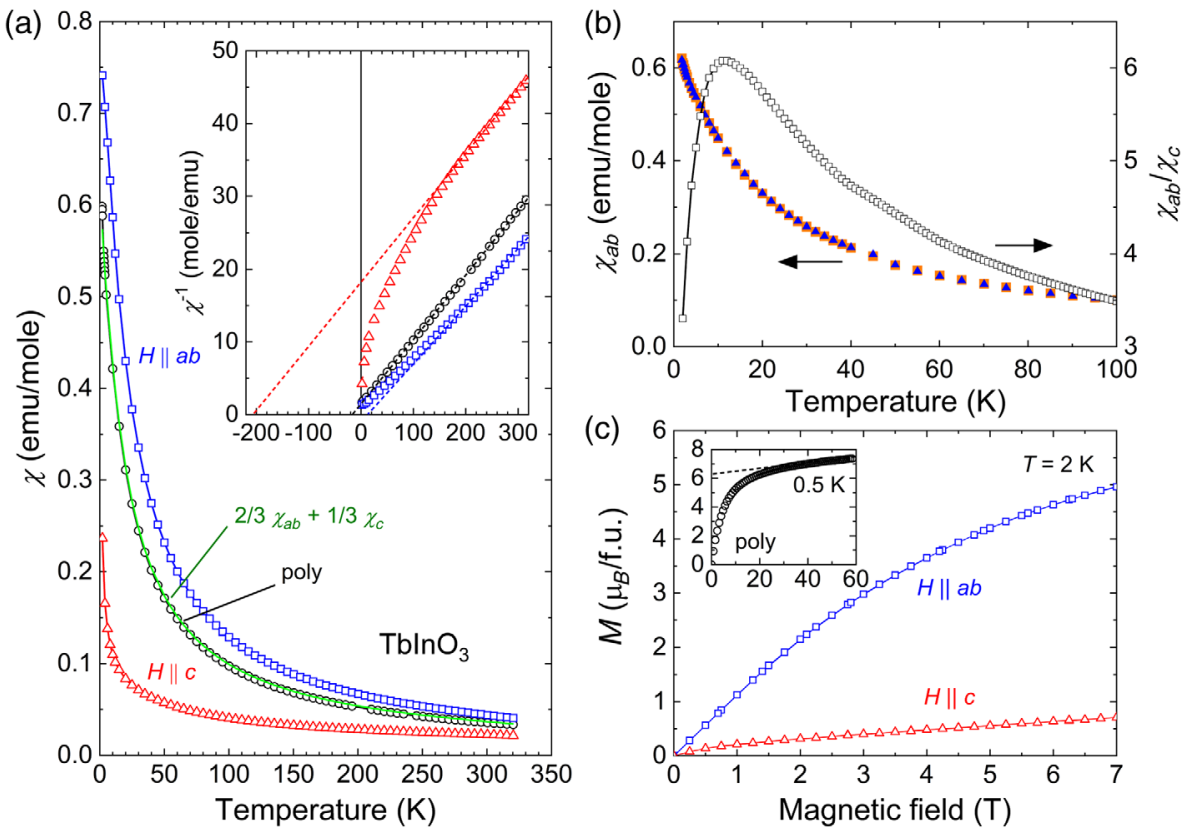

FIG. 3. (a) $\chi(T)$ of $\mathrm{TbInO}_{3}$ measured in an applied field of $\mu_{0} H=0.2 \mathrm{~T}$ after zero-magnetic-field cooling (ZFC). Black, blue, and red circles indicate data taken by using a polycrystal, a single crystal along the $a b$ plane, and the $c$ axis, respectively. The green solid line shows the averaged $\chi(T)$ curve based on the single-crystal data as explained in the text. The inset in (a) shows the $\chi^{-1}(T)$ of the polycrystal. (b) (Left) In-plane $\chi(T)$ of $\mathrm{TbInO}_{3}$ under $5 \mathrm{mT}$. Blue triangles and orange circles denote data during warming runs after $\mathrm{ZFC}$ and FC, respectively. (Right) Anisotropy ratio $\left(\chi_{a b} / \chi_{c}\right)$ as a function of the temperature. (c) Magnetization versus magnetic field at $2.0 \mathrm{~K}$. The inset shows the polycrystal data measured up to $60 \mathrm{~T}$ at $0.5 \mathrm{~K}$ by using a pulsed magnet. The dashed line in the inset is a linear fit to the data above $40 \mathrm{~T}$. 

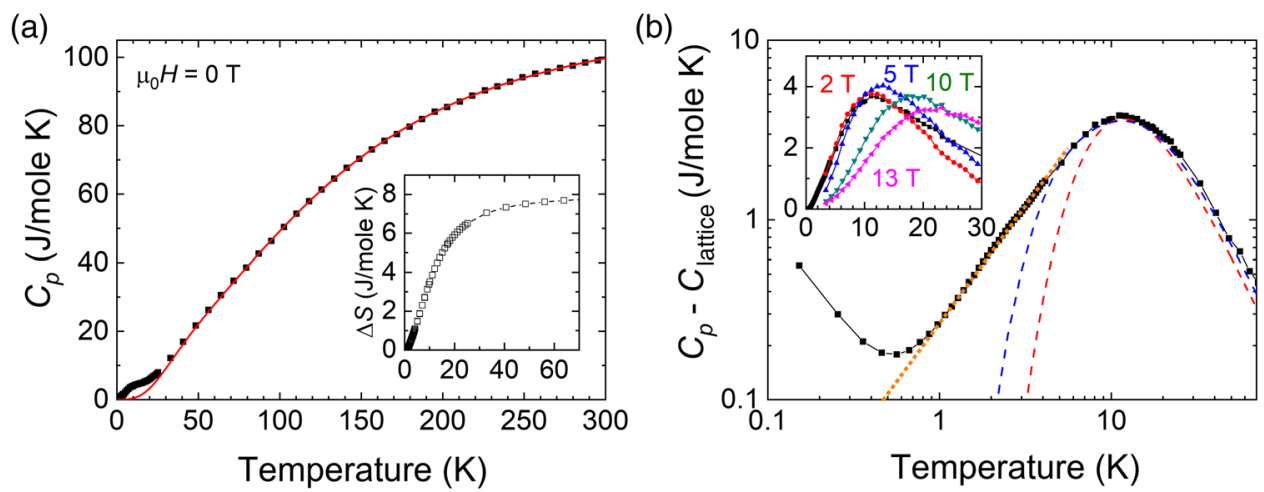

FIG. 4. (a) $C_{p}(T)$ for polycrystalline $\mathrm{TbInO}_{3}$. The red line denotes the lattice contribution described in the text. The inset shows the magnetic entropy $(\Delta S)$ acquired by integrating the $C_{\text {res }} / T$ versus $T$ curve above $1 \mathrm{~K}$. (b) The $C_{\text {res }}$ curve in the log-log scale. Red and blue dashed lines are a fit to the Schottky model described in the text. The orange dotted linear line is a guide to the eye that follows a power law $T^{1.25}$. The inset shows the field-dependent data.

Also, there is no difference between two curves measured after magnetic-field cooling and zero-field cooling at a low magnetic field of $\mu_{0} H=5 \mathrm{mT}$ [Fig. 3(b)], ruling out any spin freezing down to $1.8 \mathrm{~K}$. Interestingly, the anisotropy ratio $\left(\chi_{a b} / \chi_{c}\right)$ increases upon cooling with a maximum of 6.0 at $T \approx 12 \mathrm{~K}$ and then decreases quickly upon further cooling. Magnetic anisotropy is also evident in fielddependent magnetization [Fig. 3(c)]. Magnetization along both directions increases monotonically up to $7 \mathrm{~T}$. Data taken for a polycrystal specimen at $T=0.5 \mathrm{~K}$ up to $60 \mathrm{~T}$ [inset in Fig. 3(c)] show no hint of field-induced phase transitions [53].

A recent $a b$ initio work on $\mathrm{TbInO}_{3}$ calculates the energy scale of the nearest-neighbor AFM spin exchange interaction as 1.1 and $0.66 \mathrm{~K}$ for $\mathrm{Tb} 2-\mathrm{Tb} 2$ and $\mathrm{Tb} 1-\mathrm{Tb} 2$ pairs, respectively [54]. The discrepancy between the theoretically predicted energy scale and experimental results (e.g., $T_{\mathrm{CW}}$ ) comes from the facts that (i) the theory assumes a magnetically ordered ground state and (ii) there exists a strong magnetic anisotropy in $\mathrm{TbInO}_{3}$.

\section{Specific heat}

The lack of a phase transition or spin freezing and large magnetic anisotropy in the $2 d$-TL $\mathrm{TbInO}_{3}$ can be consistent with a spin liquid state. To confirm the absence of any phase transition at lower temperatures, we perform specific heat $\left(C_{p}\right)$ measurements on a polycrystal sample down to $0.15 \mathrm{~K}$. Figure 4(a) shows the $C_{p}(T)$ curve in a zero field. Three features exist in different temperature regions. At $T>100 \mathrm{~K}, C_{p}$ is dominated by the lattice contribution and can be fitted to a Debye model with two Debye temperatures: 190 and $630 \mathrm{~K}$ (red line). Figure 4(b) shows the residual specific heat $\left(C_{\mathrm{res}}=C_{p}-C_{\text {lattice }}\right)$ after subtracting the lattice contribution in a log-log scale. For an intermediate temperature range $1 \mathrm{~K}<T<20 \mathrm{~K}$, a hump appears with a maximum around $T=12 \mathrm{~K}$, which is from the Schottky anomaly of $\mathrm{Tb}$ electronic spins that is discussed later. At $T<0.5 \mathrm{~K}$ [Fig. 3(e)], we find an increase of $C_{\text {res }}$ upon further cooling. This feature likely originates from the nuclear Schottky anomaly of Tb and In ions $[55,56]$. Thus, at all temperatures studied, we do not find any sharp peak feature that signals a phase transition, even though the lowest temperature studied is 2 orders of magnitude smaller than $T_{\mathrm{CW}}$ obtained from the polycrystal result. Nevertheless, the nuclear Schottky anomaly that arises below $0.5 \mathrm{~K}$ limits a solid determination of the ground state of this system and warrants further investigations. In a small temperature window at $1 \mathrm{~K}<T<4 \mathrm{~K}$, there exists a large residual specific heat, which is not accounted for by the Schottky contribution from Tb electronic spins nor by that of $\mathrm{Tb}$ or In nuclear spins. In this region, $C_{\text {res }}$ follows an anomalous power-law behavior $T^{\alpha}$ with an exponent $\alpha \sim 1.25$ (orange dotted line). The $\alpha$ value for $S=1 / 22 d$-TL systems is predicted to be $2 / 3$ [57,58], experimentally observed to be 1 in organic salts [59], and is close to $2 / 3$ in $\mathrm{YbMgGaO}_{4}$ [18]. The value observed in $\mathrm{TbInO}_{3}(1.25)$ is larger than these values and needs to be studied further. We can estimate the entropy change $(\Delta S)$ of $\mathrm{Tb}$ electronic spins by calculating $\int\left(C_{\text {res }} / T\right) d T$ between 1 and $70 \mathrm{~K}$ as shown in the inset in Fig. 4(a). $\Delta S$ increases monotonically in temperature and reaches a saturation value of $7.7 \mathrm{~J} / \mathrm{mole} \cdot \mathrm{K}$ at $70 \mathrm{~K}$.

\section{DISCUSSION}

Further analysis of the Schottky anomaly centered at $12 \mathrm{~K}$ gives a better understanding of the $\mathrm{CF}$ levels of $\mathrm{Tb}$. Since $\mathrm{Tb}^{3+}$ is a non-Kramers ion with a $4 f^{8}$ electron configuration, the ground state degeneracy can be entirely lifted by the $\mathrm{CF}$, and the system can have a nonmagnetic singlet ground state. However, we find that a simple twolevel Schottky model does not properly fit to our specific heat data. Furthermore, the experimentally obtained $\Delta S$ value at $70 \mathrm{~K}(7.7 \mathrm{~J} / \mathrm{mole} \cdot \mathrm{K})$ significantly deviates from the expected $\Delta S$ value for a two-level system with the same 
degeneracy between the ground and first excited state $(5.76 \mathrm{~J} / \mathrm{mole} \cdot \mathrm{K})$ and for a two-level system with a singlet ground state and a doublet excited state $(9.13 \mathrm{~J} / \mathrm{mole} \cdot \mathrm{K})$.

In this regard, it is necessary to consider two different site symmetries in $\mathrm{TbInO}_{3}$ and allow them to have different $\mathrm{CF}$ level schemes. For simplicity, we limit the discussion to two levels that can be either a singlet or a doublet and analyze our specific heat data with a modified two-level Schottky model considering (i) two different $\mathrm{Tb}$ sites with a 1:2 ratio and (ii) degeneracy as in the following equation [60]:

$$
\begin{aligned}
C_{\text {mag }}= & \frac{1}{3} R\left(\frac{\Delta_{\mathrm{Tb} 1}}{T}\right)^{2} \frac{\left(\frac{g_{01}}{g_{11}}\right) \exp \left(\frac{\Delta_{\mathrm{Tb} 1}}{T}\right)}{\left[1+\left(\frac{g_{01}}{g_{11}}\right) \exp \left(\frac{\Delta_{\mathrm{Tb} 1}}{T}\right)\right]^{2}} \\
& +\frac{2}{3} R\left(\frac{\Delta_{\mathrm{Tb} 2}}{T}\right)^{2} \frac{\left(\frac{g_{02}}{g_{12}}\right) \exp \left(\frac{\Delta_{\mathrm{Tb} 2}}{T}\right)}{\left[1+\left(\frac{g_{02}}{g_{12}}\right) \exp \left(\frac{\Delta_{\mathrm{Tb} 2}}{T}\right)\right]^{2}} .
\end{aligned}
$$

Here, $\Delta_{\mathrm{Tb} i}, g_{0 i}$, and $g_{1 i}$ denote the energy difference between the ground state and the excited state, the degeneracy of the ground state, and the excited state, respectively. $i$ denotes either $\mathrm{Tb} 1$ or $\mathrm{Tb} 2$, and $R$ is the gas constant. The $g$ value equals the degeneracy of the corresponding state.

First, we apply a simple two-level system $\left(g_{0 i}=g_{1 i}\right)$ with $\Delta_{\mathrm{Tb} 1}=\Delta_{\mathrm{Tb} 2}=28.2 \mathrm{~K}$ [red dashed line in Fig. 4(b)]. Allowing different values of $\Delta_{T b i}$ does not yield a better result in this case. Second, we assume that $\Delta_{T b 1}=17.8 \mathrm{~K}$, $\Delta_{T b 2}=36.2 \mathrm{~K}, \mathrm{~Tb} 1$ has a singlet ground state to a doublet excited state (i.e., $g_{01} / g_{11}=0.5$ ), and $\mathrm{Tb} 2$ is a two-level system with the same degeneracy (i.e., $g_{02} / g_{12}=1$ ). This assumption greatly improves the fitting and better matches the data compared to the former [blue dashed line in Fig. 4(b)]. The magnetic entropy calculated for the latter is $6.88 \mathrm{~J} / \mathrm{mole} \mathrm{K}$, which is $90 \%$ of the experimentally obtained value at $70 \mathrm{~K}$. Fittings with different combinations of $g_{0 i}$ and $g_{1 i}$ values for $\mathrm{Tb} 1$ and $\mathrm{Tb} 2$ ions are shown in Supplemental Material [43], but none of them yield a superior fit to the data nor a proper entropy value compared to the case explained above.

Our analysis strongly points to different $\mathrm{CF}$ levels with a singlet to doublet and the same degeneracies for the ground and first excited state for $\mathrm{Tb} 1$ and $\mathrm{Tb} 2$ sites, respectively. While this analysis alone does not guarantee that the ground state of the $\mathrm{Tb} 2$ site is magnetic (i.e., doublet ground state), it is noteworthy to mention that the $\Delta_{T b 2}$ value obtained from the fit $(36.2 \mathrm{~K})$ is larger than the $T_{\mathrm{CW}}=-20.6 \mathrm{~K}$ from the polycrystal magnetic susceptibility measurement. If the ground state of $\mathrm{Tb} 2$ were to be nonmagnetic, there should be a reduction of the effective magnetic moment below $36.2 \mathrm{~K}$, which is absent in our data. Thus, our bulk features provide strong supporting evidence that only $\mathrm{Tb} 2$ has a magnetic ground state and forms a $2 d$-honeycomb magnetic lattice, consistent with the previous inelastic neutron scattering [36]. We note, however, that our model shows larger energy gaps in both
$\mathrm{Tb}$ sites and different first excited state degeneracy (doublet) in the $\mathrm{Tb} 2$ site compared to their result. This discrepancy may stem from the subtle dependence in fitting the neutron spectra possibly due to $\mathrm{Tb}-\mathrm{Tb}$ correlation [36], which requires further studies.

The Schottky anomaly in $\mathrm{TbInO}_{3}$ exhibits a magnetic field dependence that also support the above interpretation. The inset in Fig. 4(b) shows the $C_{\text {res }}(T)$ curves under different magnetic fields. At $2 \mathrm{~T}$, the broad maximum position is identical, but the high-temperature tail shows a reduced value compared to that of the zero field. At $\mu_{0} H \geq 5 \mathrm{~T}$, the hump broadens, and its maximum position shifts to a higher temperature. This behavior is typically observed in systems where a doublet state splits in energy by the Zeeman effect and the energy difference between different levels increases further on an increase in the applied magnetic field. The nonlinearity of the broad maximum versus the applied magnetic field may come from the fact that the measurement is done with a polycrystal or there exist level crossings at low fields.

High-field magnetization data also support the sitedependent ground states in $\mathrm{TbInO}_{3}$. The inset in Fig. 3(d) shows the $M(H)$ curve of the polycrystalline sample up to $60 \mathrm{~T}$ measured at $0.5 \mathrm{~K}$. The magnetization becomes linear above $40 \mathrm{~T}$, perhaps due to the van Vleck paramagnetism of $\mathrm{Tb}$ ions. The saturation magnetization after subtracting this linear term is $6.2 \mu_{B} / \mathrm{Tb}^{3+}$. This value is approximately $2 / 3$ of the free $\mathrm{Tb}^{3+}$ spin $\left(6.48 \mu_{B}\right)$ and consistent with the occupation ratio of nonmagnetic $\mathrm{Tb} 1$ and magnetic $\mathrm{Tb} 2$ in $\mathrm{TbInO}_{3}$.

Overall, our analysis suggests that $\mathrm{TbInO}_{3}$ realizes a $2 d$-honeycomb structure and a spin liquid behavior, which is of significant interest regarding the anisotropic bonddependent Kitaev interaction and Majorana modes in the spin liquid phase [61-64]. We also note that, even if all $\mathrm{Tb}$ ions are magnetic, recent theoretical studies claim that a bond-dependent exchange interaction is allowed by symmetry in $2 d-\mathrm{TL}$ and can be mapped to a Kitaev-type Hamiltonian [21-23].

Our magnetic data on polycrystal and an averaged single crystal are identical even though the length of domain boundaries in each case varies from the order of $100 \mathrm{~nm}$ (polycrystal) to $10 \mu \mathrm{m}$ (single crystal) as shown in our TEM and PFM images. In addition, there is no spin freezing or LRO down to $0.1 \mathrm{~K}$ in muon spin relaxation work on our polycrystalline sample with dense FE boundaries present [36]. These observations strongly imply that FE domain boundaries do not act as a source of disorder, because it is not a random but a deterministic structural distortion. Structural distortions may become important at low temperatures, which require further studies to elucidate how the possible spin liquid state is affected.

On the other hand, large domains and atomically sharp domain boundaries in $\mathrm{TbInO}_{3}$ offer an unprecedented opportunity to study mesoscopic edge states in a spin 


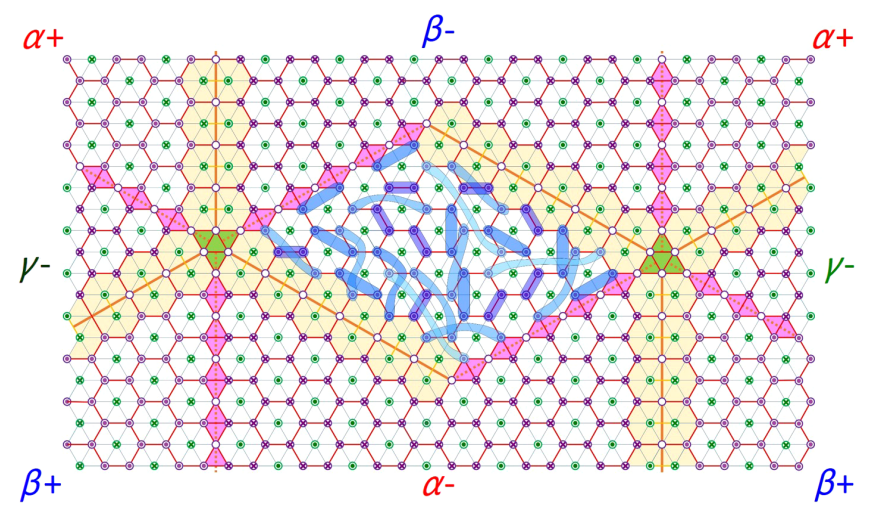

FIG. 5. A schematic in-plane structure of $\mathrm{TbInO}_{3}$ describing the structural and ferroelectric domains and domain walls. The innermost region $(\gamma+)$ shows a snapshot of entangled spins (blue loops) in the spin liquid state. Other domains are shown as $(\alpha, \beta$, $\gamma,+,-)$, and they are delineated by domain walls with full and dashed orange lines, respectively. Only $\mathrm{Tb} 1$ and $\mathrm{Tb} 2$ ions are shown as green and purple circles, respectively. Dot and crossed circles denote the up and down displacements of $\mathrm{Tb}$ ions along the $c$ axis, respectively. Blue and red lines are nearest-neighbor $\mathrm{Tb} 1-\mathrm{Tb} 2$ and $\mathrm{Tb} 2-\mathrm{Tb} 2$ bonds, respectively. There are two types of domain boundaries: a corner-sharing type (lines of magenta parallelograms) and an elongated type (lines of light yellow honeycombs).

liquid material with real spatial resolution [34]. Figure 5 schematically illustrates a snapshot of a spin liquid state that is contained within a structural domain (in this case, $\gamma+$ ). Four atomically sharp structural domain walls and two vortex cores surround the $\gamma+$ domain. The magnetic exchange paths across these boundaries are different from that within a domain, since the connectivity between $\mathrm{Tb}$ ions is modified at structural domain walls or vortex cores. Exchange interactions along the $\mathrm{Tb}-\mathrm{Tb}$ bonds are modified along these domain boundaries, similar to lattice defects in a Kitaev honeycomb lattice, and may host unconventional magnetic states [37-39].

In addition to the general expectations from a spin liquid, $\mathrm{TbInO}_{3}$ also provides further functionalities in analogy to $h-R \mathrm{MnO}_{3}$ systems [65-67]. The FE domain boundaries can be electrically charged in the plane containing the $c$ axis, and these charged walls may accompany mobile electrical carriers, which is generally difficult to realize in other insulating spin liquid systems [68].

\section{CONCLUSION}

Hexagonal $\mathrm{TbInO}_{3}$ hosts two exotic examples of ferroelectricity and magnetism: topological ferroelectric vortices and spin liquid behavior. Our bulk data suggest that $\mathrm{TbInO}_{3}$ has an in-plane magnetic anisotropy without any LRO down to $0.15 \mathrm{~K}$ with the emergence of a $2 d$-honeycomb magnetic structure. The existence of well-defined, atomically sharp ferroelectric domain boundaries in this system may open a new avenue for seeking topological edge states in spin liquids that has not been studied to date. Further studies are necessary to understand the exact spin liquid nature and to probe locally the possible nontrivial edge state in this system.

\section{ACKNOWLEDGMENTS}

We thank V. Kiryukhin, M. G. Kim, and J. Y. Kim for helpful discussions. The work at Rutgers was supported by the Gordon and Betty Moore Foundation's EPiQS Initiative through Grant No. GBMF4413 to the Rutgers Center for Emergent Materials. The NHMFL Pulsed Field Facility is supported by the NSF, the U.S. DOE, and the State of Florida through NSF cooperative Grants No. DMR1157490 and DMR-1644779. Work at Los Alamos National Laboratory was supported by the LANL LDRD program.

[1] A. P. Ramirez, Strongly Geometrically Frustrated Magnets, Annu. Rev. Mater. Sci. 24, 453 (1994).

[2] M. F. Collins and O. A. Petrenko, Triangular Antiferromagnets, Can. J. Phys. 75, 605 (1997).

[3] G. Wannier, Antiferromagnetism. The Triangular Ising Net, Phys. Rev. 79, 357 (1950).

[4] K. Husimi and I. Syoji, The Statistics of Honeycomb and Triangular Lattice. I., Prog. Theor. Phys. 5, 177 (1950).

[5] H. Kawamura and S. Miyashita, Phase Transition of the Two-Dimensional Heisenberg Antiferromagnet on the Triangular Lattice, J. Phys. Soc. Jpn. 53, 4138 (1984).

[6] L. Seabra, T. Momoi, P. Sindzingre, and N. Shannon, Phase Diagram of the Classical Heisenberg Antiferromagnet on a Triangular Lattice in an Applied Magnetic Field, Phys. Rev. B 84, 214418 (2011).

[7] P. W. Anderson, Resonating Valence Bonds: A New Kind of Insulator?, Mater. Res. Bull. 8, 153 (1973).

[8] F. Mila, Quantum Spin Liquids, Eur. J. Phys. 21, 499 (2000).

[9] L. Balents, Spin Liquids in Frustrated Magnets, Nature (London) 464, 199 (2010).

[10] L. Savary and L. Balents, Quantum Spin Liquids: A Review, Rep. Prog. Phys. 80, 016502 (2017).

[11] Y. Zhou, K. Kanoda, and T.-K. Ng, Quantum Spin Liquid States, Rev. Mod. Phys. 89, 025003 (2017).

[12] B. Bernu, C. Lhuillier, and L. Pierre, Signature of Néel Order in Exact Spectra of Quantum Antiferromagnets on Finite Lattices, Phys. Rev. Lett. 69, 2590 (1992).

[13] L. Capriotti, A. E. Trumper, and S. Sorella, Long-Range Néel Order in the Triangular Heisenberg Model, Phys. Rev. Lett. 82, 3899 (1999).

[14] S. R. White and A. L. Chernyshev, Neél Order in Square and Triangular Lattice Heisenberg Models, Phys. Rev. Lett. 99, 127004 (2007).

[15] S. T. Bramwell and M. J. P. Gingras, Spin Ice State in Frustrated Magnetic Pyrochlore Materials, Science 294, 1495 (2001).

[16] J. S. Gardner, M. J. P. Gingras, and J. E. Greedan, Magnetic Pyrochlore Oxides, Rev. Mod. Phys. 82, 53 (2010). 
[17] M. R. Norman, Colloquium: Herbertsmithite and the Search for the Quantum Spin Liquid, Rev. Mod. Phys. 88, 041002 (2016).

[18] Y. Li, H. Liao, Z. Zhang, S. Li, F. Jin, L. Ling, L. Zhang, Y. Zou, L. Pi, Z. Yang, J. Wang, Z. Wu, and Q. Zhang, Gapless Quantum Spin Liquid Ground State in the Two-Dimensional Spin-1/2 Triangular Antiferromagnet $\mathrm{YbMgGaO}_{4}$, Sci. Rep. 5, 16419 (2015).

[19] Y. Li, G. Chen, W. Tong, L. Pi, J. Liu, Z. Yang, X. Wang, and Q. Zhang, Rare-Earth Triangular Lattice Spin Liquid: A Single-Crystal Study of $\mathrm{YbMgGaO}_{4}$, Phys. Rev. Lett. 115, 167203 (2015).

[20] Y.-D. Li, X. Wang, and G. Chen, Hidden Multipolar Orders of Dipole-Octupole Doublets on a Triangular Lattice, Phys. Rev. B 94, 201114(R) (2016).

[21] Y.-D. Li, X. Wang, and G. Chen, Anisotropic Spin Model of Strong Spin-Orbit-Coupled Triangular Antiferromagnets, Phys. Rev. B 94, 035107 (2016).

[22] C. Liu, Y.-D. Li, and G. Chen, Selective Measurements of Intertwined Multipolar Orders: Non-Kramers Doublets on a Triangular Lattice, Phys. Rev. B 98, 045119 (2018).

[23] Z. Zhu, P. A. Maksimov, S. R. White, and A. L. Chernyshev, Topography of Spin Liquids on a Triangular Lattice, Phys. Rev. Lett. 120, 207203 (2018).

[24] Y. Li, D. Adroja, R. I. Bewley, D. Voneshen, A. A. Tsirlin, P. Gegenwart, and Q. Zhang, Crystalline Electric-Field Randomness in the Triangular Lattice Spin-Liquid $\mathrm{YbMgGaO}_{4}$, Phys. Rev. Lett. 118, 107202 (2017).

[25] Z. Zhu, P. A. Maksimov, S. R. White, and A. L. Chernyshev, Disorder-Induced Mimicry of a Spin Liquid in $\mathrm{YbMgGaO}_{4}$, Phys. Rev. Lett. 119, 157201 (2017).

[26] M. B. Sanders, F. A. Cevallos, and R. J. Cava, Magnetism in the $\mathrm{KBaRE}\left(\mathrm{BO}_{3}\right)_{2}(\mathrm{RE}=\mathrm{Sm}, \mathrm{Eu}, \mathrm{Gd}, \mathrm{Tb}, \mathrm{Dy}, \mathrm{Ho}, \mathrm{Er}, \mathrm{Tm}$, $Y b$, Lu) Series: Materials with a Triangular Rare Earth Lattice, Mater. Res. Express 4, 036102 (2017).

[27] X. G. Wen, Gapless Boundary Excitations in the Quantum Hall States and in the Chiral Spin States, Phys. Rev. B 43, 11025 (1991).

[28] H. Katsura, N. Nagaosa, and P. A. Lee, Theory of the Thermal Hall Effect in Quantum Magnets, Phys. Rev. Lett. 104, 066403 (2010).

[29] M. Hirschberger, J. W. Krizan, R. J. Cava, and N. P. Ong, Large Thermal Hall Conductivity of Neutral Spin Excitations in a Frustrated Quantum Magnet, Science 348, 106 (2015).

[30] Y. Kasahara, T. Ohnishi, Y. Mizukami, O. Tanaka, S. Ma, K. Sugii, N. Kurita, H. Tanaka, J. Nasu, Y. Motome, T. Shibauchi, and Y. Matsuda, Majorana Quantization and Half-Integer Thermal Quantum Hall Effect in a Kitaev Spin Liquid, Nature (London) 559, 227 (2018).

[31] K. von Klitzing, The Quantized Hall Effect, Rev. Mod. Phys. 58, 519 (1986).

[32] L. Ju, Z. Shi, N. Nair, Y. Lv, C. Jin, J. Velasco, C. OjedaAristizabal, H. A. Bechtel, M. C. Martin, A. Zettl, J. Analytis, and F. Wang, Topological Valley Transport at Bilayer Graphene Domain Walls, Nature (London) 520, 650 (2015).

[33] Y. Feng, X. Feng, Y. Ou, J. Wang, C. Liu, L. Zhang, D. Zhao, G. Jiang, S.-C. Zhang, K. He, X. Ma, Q.-K. Xue, and Y. Wang, Observation of the Zero Hall Plateau in a
Quantum Anomalous Hall Insulator, Phys. Rev. Lett. 115, 126801 (2015).

[34] J. Knolle and R. Moessner, A Field Guide to Spin Liquids, Annu. Rev. Condens. Matter Phys. 10, 451 (2019).

[35] S.C. Abrahams, Ferroelectricity and Structure in the $\mathrm{YMnO}_{3}$ Family, Acta Crystallogr. Sect. B 57, 485 (2001).

[36] L. Clark, G. Sala, D. D. Maharaj, M. B. Stone, K. S. Knight, M. T. F. Telling, X. Wang, X. Xu, J. Kim, Y. Li, S.-W. Cheong, and B. D. Gaulin, Two-Dimensional Spin Liquid Behaviour in the Triangular-Honeycomb Antiferromagnet $\mathrm{TbInO}_{3}$, Nat. Phys. 15, 262 (2019).

[37] O. Petrova, P. Mellado, and O. Tchernyshyov, Unpaired Majorana Modes on Dislocations and String Defects in Kitaev's Honeycomb Model, Phys. Rev. B 90, 134404 (2014).

[38] J. Brennan and J. Vala, Lattice Defects in the Kitaev Honeycomb Model, J. Phys. Chem. A 120, 3326 (2016).

[39] H. Wang, H. J. Changlani, Y. Wan, and O. Tchernyshyov, Quantum Spin Liquid with Seven Elementary Particles, Phys. Rev. B 95, 144425 (2017).

[40] J. A. Detwiler, G. M. Schmiedeshoff, N. Harrison, A. H. Lacerda, J. C. Cooley, and J. L. Smith, Magnetization of $\mathrm{UBe}_{13}$ to 60 T, Phys. Rev. B 61, 402 (2000).

[41] B. Paul, S. Chatterjee, S. Gop, A. Roy, V. Grover, R. Shukla, and A. K. Tyagi, Evolution of Lattice Dynamics in Ferroelectric Hexagonal $\mathrm{REInO}_{3}(\mathrm{RE}=\mathrm{Ho}, \mathrm{Dy}, \mathrm{Tb}, \mathrm{Gd}, \mathrm{Eu}, \mathrm{Sm})$ Perovskites, Mater. Res. Express 3, 075703 (2016).

[42] T. Tohei, H. Moriwake, H. Murata, A. Kuwabara, R. Hashimoto, T. Yamamoto, and I. Tanaka, Geometric Ferroelectricity in Rare-Earth Compounds $\mathrm{RGaO}_{3}$ and $\mathrm{RInO}_{3}$, Phys. Rev. B 79, 144125 (2009).

[43] See Supplemental Material at http://link.aps.org/ supplemental/10.1103/PhysRevX.9.031005 for detailed comparison between different degeneracy combinations and structural refinement parameters.

[44] B. B. Van Aken, T. T. M. Palstra, A. Filippetti, and N. A. Spaldin, The Origin of Ferroelectricity in Magnetoelectric $\mathrm{YMnO}_{3}$, Nat. Mater. 3, 164 (2004).

[45] C. J. Fennie and K. M. Rabe, Ferroelectric Transition in $\mathrm{YMnO}_{3}$ from First Principles, Phys. Rev. B 72, 100103(R) (2005).

[46] T. Choi, Y. Horibe, H. T. Yi, Y. J. Choi, W. Wu, and S.-W. Cheong, Insulating Interlocked Ferroelectric and Structural Antiphase Domain Walls in Multiferroic $\mathrm{YMnO}_{3}$, Nat. Mater. 9, 253 (2010).

[47] T. Katsufuji, M. Masaki, A. Machida, M. Moritomo, K. Kato, E. Nishibori, M. Takata, M. Sakata, K. Ohoyama, K. Kitazawa, and H. Takagi, Crystal Structure and Magnetic Properties of Hexagonal $\mathrm{RMnO}_{3}(R=Y, L u$, and $S c)$ and the Effect of Doping, Phys. Rev. B 66, 134434 (2002).

[48] F.-T. Huang and S.-W. Cheong, Aperiodic Topological Order in the Domain Configurations of Functional Materials, Nat. Rev. Mater. 2, 17004 (2017).

[49] S. C. Chae, Y. Horibe, D. Y. Jeong, S. Rodan, N. Lee, and S.-W. Cheong, Self-Organization, Condensation, and Annihilation of Topological Vortices and Antivortices in a Multiferroic, Proc. Natl. Acad. Sci. U.S.A. 107, 21366 (2010).

[50] S.-Z. Lin, X. Wang, Y. Kamiya, G.-W. Chern, F. Fan, D. Fan, B. Casas, Y. Liu, V. Kiryukhin, W. H. Zurek, C. D. 
Batista, and S.-W. Cheong, Topological Defects as Relics of Emergent Continuous Symmetry and Higgs Condensation of Disorder in Ferroelectrics, Nat. Phys. 10, 970 (2014).

[51] X. Wang, F.-T. Huang, R. Hu, F. Fan, and S.-W. Cheong, Self-Poling with Oxygen Off-Stoichiometry in Ferroelectric Hexagonal Manganites, APL Mater. 3, 041505 (2015).

[52] N. Fujimura, T. Ishida, T. Yoshimura, and T. Ito, Epitaxially Grown $\mathrm{YMnO}_{3}$ Film: New Candidate for Nonvolatile Memory Devices, Appl. Phys. Lett. 69, 1011 (1996).

[53] P. Chen, B. S. Holinsworth, K. R. O’Neal, T. V. Brinzari, D. Mazumdar, C. V. Topping, X. Luo, S.-W. Cheong, J. Singleton, S. McGill, and J. L. Musfeldt, Magnetochromic Effect in Multiferroic $\mathrm{RIn}_{1-x} \mathrm{Mn}_{x} \mathrm{O}_{3}(R=\mathrm{Tb}, D y)$, Phys. Rev. B 91, 205130 (2015).

[54] E. E. Gordon, X. Cheng, J. Kim, S.-W. Cheong, S. Deng, and M.-H. Whangbo, Nonequivalent Spin Exchanges of the Hexagonal Spin Lattice Affecting the Low-Temperature Magnetic Properties of $\mathrm{RInO}_{3}(R=\mathrm{Gd}, T b, D y)$ : Importance of Spin-Orbit Coupling for Spin Exchanges between Rare-Earth Cations with Nonzero Orbital Moments, Inorg. Chem. 57, 9260 (2018).

[55] R. W. Hill, The Specific Heats of $\mathrm{Tb}_{2} \mathrm{O}_{3}$ and between 0.5 and 22 K, J. Phys. C 19, 673 (1986).

[56] B. Bleaney and R. W. Hill, Hyperfine Structure in Terbium Metal, Proc. Phys. Soc. 78, 313 (1961).

[57] O. I. Motrunich, Variational Study of Triangular Lattice Spin-1/2 Model with Ring Exchanges and Spin Liquid State in $\kappa-(\mathrm{ET})_{2} \mathrm{Cu}_{2}(\mathrm{CN})_{3}$, Phys. Rev. B 72, 045105 (2005).

[58] S.-S. Lee and P. A. Lee, U(1) Gauge Theory of the Hubbard Model: Spin Liquid States and Possible Application to $\kappa$-(BEDT-TTF $)_{2} \mathrm{Cu}_{2}(\mathrm{CN})_{3}$, Phys. Rev. Lett. 95, 036403 (2005).

[59] S. Yamashita, T. Yamamoto, Y. Nakazawa, M. Tamura, and R. Kato, Gapless Spin Liquid of an Organic Triangular Compound Evidenced by Thermodynamic Measurements, Nat. Commun. 2, 275 (2011).
[60] A. Tari, The Specific Heat of Matter at Low Temperatures (Imperial College, London, 2003).

[61] K. W. Plumb, J. P. Clancy, L. J. Sandilands, V. V. Shankar, Y. F. Hu, K. S. Burch, H.-Y. Kee, and Y.-J. Kim, $\alpha-\mathrm{RuCl}_{3}$ : $A$ Spin-Orbit Assisted Mott Insulator on a Honeycomb Lattice, Phys. Rev. B 90, 041112(R) (2014).

[62] S. H. Chun, J.-W. Kim, J. Kim, H. Zheng, C. C. Stoumpos, C. D. Malliakas, J. F. Mitchell, K. Mehlawat, Y. Singh, Y. Choi, T. Gog, A. Al-Zein, M. M. Sala, M. Krisch, J. Chaloupka, G. Jackeli, G. Khaliullin, and B. J. Kim, Direct Evidence for Dominant Bond-Directional Interactions in a Honeycomb Lattice Iridate $\mathrm{Na}_{2} \mathrm{IrO}_{3}$, Nat. Phys. 11, 462 (2015).

[63] A. Banerjee, C. A. Bridges, J.-Q. Yan, A. A. Aczel, L. Li, M. B. Stone, G. E. Granroth, M. D. Lumsden, Y. Yiu, J. Knolle, S. Bhattacharjee, D. L. Kovrizhin, R. Moessner, D. A. Tennant, D. G. Mandrus, and S. E. Nagler, Proximate Kitaev Quantum Spin Liquid Behaviour in a Honeycomb Magnet, Nat. Mater. 15, 733 (2016).

[64] S.-H. Do, S.-Y. Park, J. Yoshitake, J. Nasu, Y. Motome, Y. S. Kwon, D. T. Adroja, D. J. Voneshen, K. Kim, T.-H. Jang, J.-H. Park, K.-Y. Choi, and S. Ji, Majorana Fermions in the Kitaev Quantum Spin System $\alpha-\mathrm{RuCl}_{3}$, Nat. Phys. 13, 1079 (2017).

[65] D. Meier, Functional Domain Walls in Multiferroics, J. Phys. Condens. Matter 27, 463003 (2015).

[66] D. Meier, J. Seidel, A. Cano, K. Delaney, Y. Kumagai, M. Mostovoy, N. A. Spaldin, R. Ramesh, and M. Fiebig, Anisotropic Conductance at Improper Ferroelectric Domain Walls, Nat. Mater. 11, 284 (2012).

[67] W. Wu, Y. Horibe, N. Lee, S.-W. Cheong, and J. R. Guest, Conduction of Topologically Protected Charged Ferroelectric Domain Walls, Phys. Rev. Lett. 108, 077203 (2012).

[68] Z. A. Kelly, M. J. Gallagher, and T. M. McQueen, Electron Doping a Kagome Spin Liquid, Phys. Rev. X 6, 041007 (2016). 